\title{
Construção de um novo instrumento orientador para o exame físico respiratório de equinos
}

\section{Building a new guiding instrument for the physical respiratory examination of horses}

1 Pontifícia Universidade Católica do Paraná (PUCPR), Programa de Pós Graduação em Ciência Animal, Curitiba, PR, Brasil

2 Pontifícia Universidade Católica do Paraná (PUCPR), Programa de Pós Graduação em Tecnologia em Saúde, Curitiba, PR, Brasil

*Correspondência: tcosanti@gmail.com

Submetido: 14 nov 2020 | Aprovado: 26 mai 2021

DOI: http://dx.doi.org/10.7213/acad.2021.19005

Rev. Acad. Ciênc. Anim. 2021;19:e19005

\section{Resumo}

O diagnóstico de doenças respiratórias é desafiador aos médicos veterinários, pois os mesmos sinais clínicos são encontrados em diferentes doenças. $O$ presente estudo objetivou o aprimoramento de um instrumento orientador ao exame clínico respiratório de equinos, com a hipótese de que auxiliará no trabalho médico veterinário. Neste estudo de coorte, 19 médicos veterinários (MV) atuantes em medicina equina aceitaram participar do estudo, sendo divididos em dois grupos: MV generalistas (GMVG, $\mathrm{n}=16$ ), com profissionais graduados a menos
Thasla de Freitas Santi (i) ${ }^{1 *}$

Fernanda Cristina Mendes Barussi (D)

Fernanda Zettel Bastos (D) ${ }^{1}$

Joan Emilio Deitos ${ }^{2}$

Saulo Henrique Weber (i) ${ }^{1}$

Deborah Ribeiro Carvalho ${ }^{2}$

Pedro Vicente Michelotto Júnior (i) ${ }^{1}$ de 10 anos e MV especialistas (GMVE, $\mathrm{n}=3$ ), com mais de 10 anos de experiência profissional. Um instrumento de avaliação inicial (instrumento 1) foi disponibilizado a todos os avaliadores para ser empregado no exame físico de um equino qualquer, para em seguida responderem a um questionário a respeito da complexidade geral, preenchimento, compreensão, praticidade e tempo para preenchimento, com espaço para comentários. Com base nas respostas, um novo instrumento (2) foi construído para nova avaliação pelos MV. Foi realizada a comparação das respostas obtidas pelos avaliadores para os dois instrumentos. Como resultado inicial, a avaliação dos instrumentos foi concordante entre os dois grupos, evidenciando que a utilização dos instrumentos na prática clínica independe de experiência profissional. $O$ instrumento de avaliação 2 foi considerado menos complexo $(p=0,046)$, de fácil preenchimento $(p=0,005)$ e maior compreensão ( $p=0,003)$. Assim, mesmo em se tratando de um processo a ser continuado, conseguiu-se obter um instrumento de avaliação respiratória de equinos de melhor qualidade, e que visa auxiliar o MV de campo.

Palavras-chave: Cavalos. Diagnóstico. Doenças dos cavalos. Pulmonar. 


\section{Abstract}

The diagnosis of respiratory diseases is challenging for veterinarians, as the same clinical signs are found in different diseases. The present study aimed to improve an instrument to guide the clinical respiratory examination of horses, with the hypothesis that this device could aid equine practitioners. Nineteen veterinary doctors (MV) working in equine medicine agreed to participate in this cohort study, and were divided into two groups: the generalist MV (GMVG, $\mathrm{n}=16$ ), with professionals who had graduated less than 10 years ago; and specialist MVs (GMVE, $n=3$ ), with over 10 years of professional experience. The initial evaluation instrument (instrument 1) was made available to all evaluators to be used in the physical examination of any horse. The practitioners then answered a questionnaire regarding the general complexity, completion, understanding, practicality and time to complete the procedure, with space for comments. Based on the answers, a new instrument (2) was built, to facilitate a new way to evaluate the MV. The responses obtained by the evaluators for the two instruments were compared. As an initial result, the assessment of the instruments was consistent between the two groups of MV, showing that the use of the instruments in clinical practice would not depend on professional experience. The assessment instrument 2 was considered less complex ( $p$ $=0.046)$, more easy to complete $(p=0.005)$ and more easy to understand $(p=0.003)$. Thus, even in the case of a process to be continued, it was possible to obtain a better quality instrument for respiratory assessment in horses, with hopes of assisting the MV field.

Keywords: Horses. Diagnosis. Horse diseases. Pulmonary.

\section{Introdução}

As doenças respiratórias representam a segunda maior causa de atendimentos em equinos, depois das afecções musculoesqueléticas (Kusano et al., 2008). Os sinais clínicos mais comuns são tosse, corrimento nasal, diminuição de rendimento desportivo e de trabalho, sons respiratórios audíveis (roncos e chiados) (Seahorn e Beadle, 1993; Hoffman, 2003; Albrecht et al., 2018) e, ainda, esforço respiratório evidente em repouso, por esforço toracoabdominal aumentado e dilatação das narinas (Léguillette et al., 2000).
A tosse está presente em aproximadamente 63\% dos casos de doenças respiratórias (Kutasi et al., 2011). Apesar de ser um sinal clínico específico para o sistema respiratório, está relacionada a diferentes doenças, especialmente das vias aéreas inferiores, doenças inflamatórias, alérgicas, infecção viral, pneumonia, pleurite, hemorragia pulmonar induzida pelo exercício, parasitas pulmonares, neoplasias pulmonares, entre outras (Christley, et al., 2001; Kutasi et al., 2011; Wilson e Laktriz, 2014; Couëtil et al., 2016; Davis, 2018).

Assim como a tosse, outros sinais clínicos envolvidos na manifestação das diferentes doenças respiratórias estarão presentes em várias delas, o que torna o diagnóstico desafiador para o médico veterinário de equinos. Em vista disto, há uma grande relevância em se realizar exames periódicos, históricos detalhados e exames complementares específicos para efetivar o diagnóstico do paciente, principalmente os de evolução assintomática (Robertson, 1991; Fernandes et al., 2011).

Desta forma, o presente estudo levantou a hipótese de que o instrumento orientador para o exame físico dos cavalos pode ser melhorado, refletindo no exame físico respiratório realizado por médicos veterinários de equinos. A presente pesquisa, portanto, objetivou avaliar e criar uma versão melhorada de um instrumento de avaliação clínica até então utilizado no exame físico de equinos, em um serviço de atendimento em uma instituição de ensino e pesquisa.

\section{Material e métodos}

A presente pesquisa se caracteriza por estudo de coorte onde investigou-se o instrumento de orientação para a avaliação clínica respiratória em equinos. $O$ projeto teve aprovação do Comitê de Ética em Pesquisa (parecer 3.271.277) e Uso de Animais (parecer 01629) da Pontifícia Universidade Católica do Paraná (PUCPR).

Dezenove médicos veterinários (MV) participaram do estudo, sendo divididos em dois grupos: MV generalistas (GMVG, $\mathrm{n}=16$ ) e $\mathrm{MV}$ experientes no exame respiratório (GMVE, $n=3$ ). Foram convidados para compor o grupo de avaliadores médicos veterinários atuantes na área de medicina equina pertencentes aos estados do 
Paraná, São Paulo e Minas Gerais. Como critérios de inclusão, os médicos veterinários atuantes na área de medicina de equinos deveriam ter menos de 10 anos de formação profissional para compor o GMVG e mais de 10 anos de formação profissional para compor o GMVE. Como critérios de exclusão, para ambos os grupos, considerou-se a indisponibilidade do uso dos dois instrumentos de avaliação clínica, seguido da resposta dos questionários de avaliação de ambos.

Todos os avaliadores foram solicitados a realizar o exame físico específico do sistema respiratório em um equino qualquer, utilizando o instrumento de avaliação 1 (Figura 1).

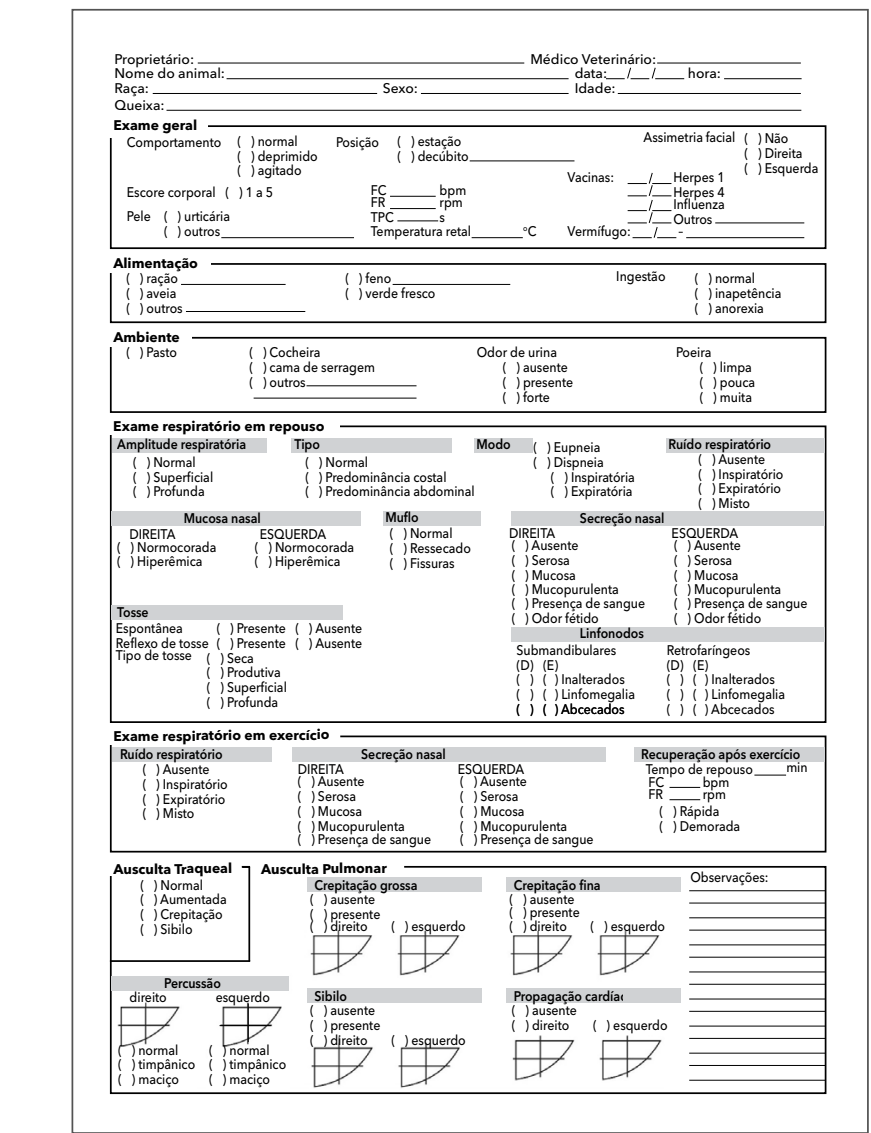

Figura 1 - Instrumento de avaliação clínica do sistema respiratório equino disponibilizado aos médicos veterinários para avaliação..

Em seguida, deveriam responder um questionário a respeito do instrumento, onde foram levantadas cinco questões quanto à complexidade dos termos, compreensão do instrumento, dificuldade, praticidade e tempo de preenchimento. Os avaliadores poderiam classificar conforme a escala em extremamente confuso, confuso, fácil e extremamente fácil, resultando em quatro diferentes níveis de compreensão. Adicionalmente, foi colocado um campo aberto para sugestões sobre o instrumento 1 em relação a possíveis melhorias ou críticas.

A partir das respostas obtidas, o instrumento de avaliação foi readequado de acordo com as opiniões dos avaliadores, com o intuito de se chegar a um instrumento melhorado, mais intuitivo e de menor complexidade para compreensão e uso, em relação ao inicial (Figuras 2 e 3). Após a alteração do instrumento, em outro momento, solicitou-se aos mesmos avaliadores que avaliassem um equino empregando o novo instrumento, denominado instrumento de avaliação 2, respondendo novamente ao questionário.

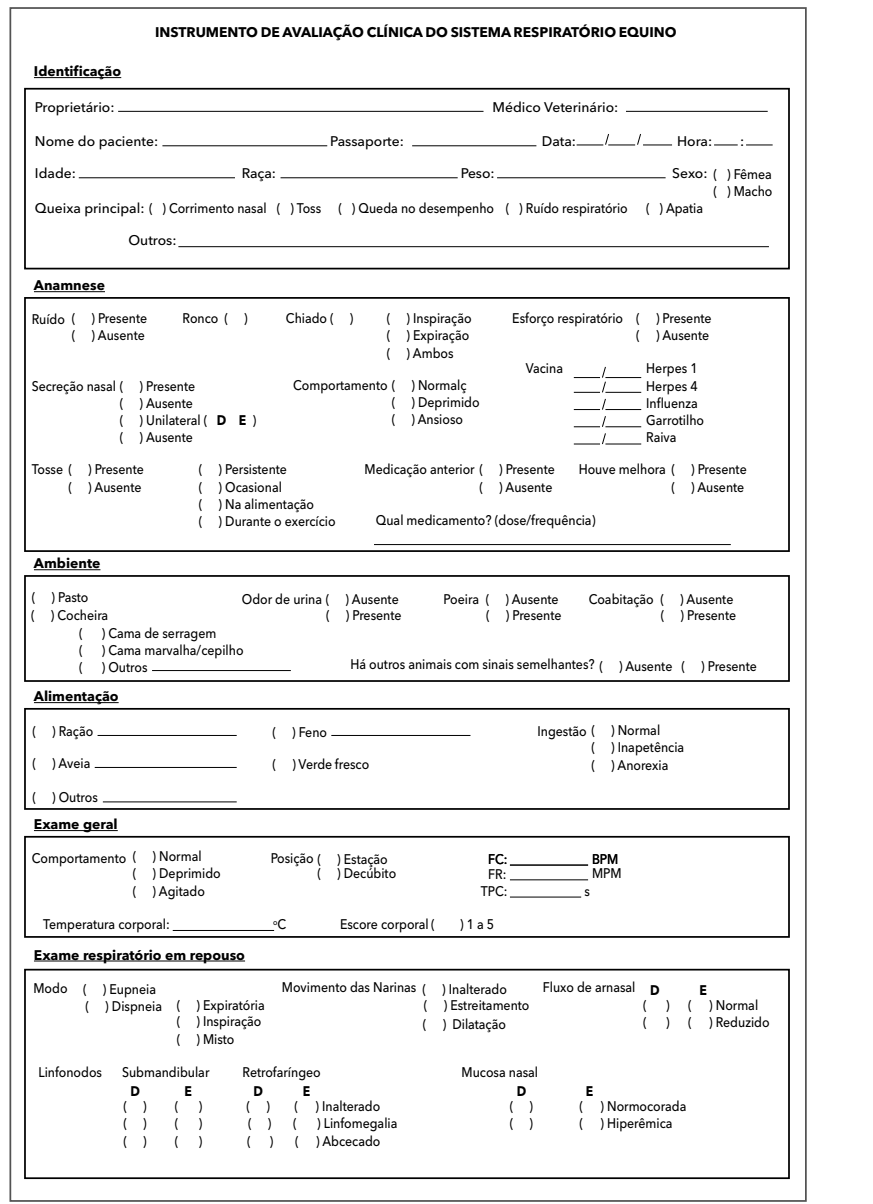

Figura 2 - Parte da frente do instrumento de avaliação clínica do sistema respiratório equino após a avaliação de médicos veterinários. 


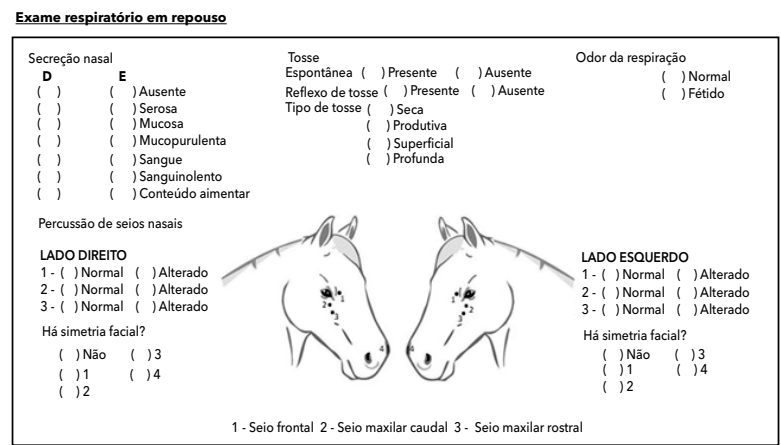

$$
\text { Ausculta }
$$

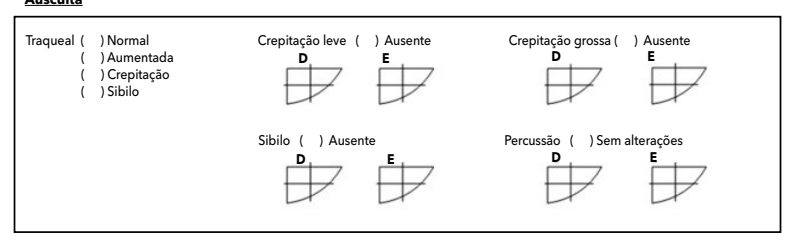

OBS:

Figura 3 - Verso do instrumento de avaliação clínica do sistema respiratório equino, após a avaliação de médicos veterinários.

A análise estatística dos dados foi feita usando o teste de Mann-Whitney para a comparação das respostas obtidas pelos avaliadores dos dois grupos, para os questionários de avaliação dos instrumentos 1 e 2. O teste de Wilcoxon foi usado para a comparação das respostas obtidas nos questionários 1 e 2 de todos os avaliadores.

Assumiu-se a concordância entre os dois grupos de avaliadores (GMVG e GMVE) através do teste de Kappa, com valores que variam de -1 até +1 e quanto mais próximo de 1 mais forte a concordância. As análises foram realizadas utilizando o programa IBM SPSS Statistics (IBM Corporation, New York, USA), considerando significativo valor de $p<0,05$.

\section{Resultados e discussão}

Dos 26 avaliadores convidados, apenas 19 participaram, sendo 9 médicos veterinários atuando em universidade no período do estudo e 10 médicos veterinários de campo. Um dos avaliadores do GMVG, o avaliador número 5, respondeu ao primeiro questionário e não se disponibilizou a responder a segunda avaliação, sendo excluído.
$\mathrm{Na}$ comparação independente entre os grupos GMVG e GMVE, para as questões a respeito do instrumento 1 de avaliação, não houve diferença entre os grupos para nenhuma das questões ( $p>$ $0,05)$ (Tabela 1). Entende-se, assim, que o primeiro instrumento serviu tanto a médicos veterinários experientes quanto a generalistas, mesmo com menor experiência na avaliação específica do sistema respiratório.

Tabela 1 - Comparação entre a avaliação do primeiro e do segundo instrumento de avaliação, com a segmentação entre os grupos de médicos veterinários generalistas (GMVG) e especialistas (GMVE), avaliando sua percepção em relação aos instrumentos

\begin{tabular}{lcc}
\hline \multirow{2}{*}{ Questões } & \multicolumn{2}{c}{ Grupos } \\
\cline { 2 - 3 } & GMVG & GMVE \\
\hline Complexidade & 0,875 & 0,875 \\
Dificuldade no preenchimento & 1,000 & 0,793 \\
Compreensão & 1,000 & 0,559 \\
Praticidade & 0,875 & 1,000 \\
Tempo de preenchimento & 1,000 & 0,171 \\
\hline
\end{tabular}

Conforme o objetivo deste estudo de se obter um instrumento de avaliação respiratória melhorado para a orientação ao exame clínico respiratório, apesar de não haver discrepância nas respostas do questionário, as sugestões dos avaliadores na forma descritiva trouxeram contribuições importantes para a melhoria do instrumento de avaliação respiratória. Desta forma, vislumbrou-se produzir um novo instrumento de avaliação clínica (Figuras 2 e 3), atendendo às necessidades levantadas para avaliar de maneira minuciosa e completa ao sistema respiratório equino. Sugestões colocadas pelos avaliadores foram implementadas com base na literatura apropriada, para construir o novo instrumento de avaliação (Wilson e Lakritz, 2014; Davis, 2018).

Em relação às questões do instrumento de avaliação 2, não houve diferença entre os grupos para nenhuma das questões $(p>0,05)$ (Tabela 1). Provavelmente o número de avaliadores por grupo influenciou esse resultado, o que certamente foi um fator limitador do estudo, mas considerado adequado estatisticamente para os objetivos do presente estudo. 
Houve concordância entre GMVG e GMVE no teste de kappa para as questões de complexidade $(\kappa=0,086)$, dificuldade $(\kappa=0,483)$, compreensão $(\kappa=0,586)$, praticidade $(\kappa=0,252)$ e tempo de preenchimento $(\kappa=0,153)$ em relação aos dois instrumentos.

Em seguida, considerou-se todos os médicos veterinários avaliadores em um único grupo, evidenciando percepção de melhora do segundo instrumento em relação ao primeiro no que diz respeito à complexidade $(p=0,046)$, facilidade no preenchimento $(p=0,005)$ e na compreensão do instrumento $(p=0,003)$. Com relação à praticidade e tempo de preenchimento, não houve mudança entre os instrumentos 1 e 2 ( $p=0,096$ e $p=0,763$, respectivamente). Com relação à praticidade, um exame clínico deve ser bastante minucioso para se chegar ao diagnóstico e, portanto, não seria adequado um instrumento que tornasse o processo menos meticuloso. Da mesma forma, a investigação clínica rigorosa exige tempo, e nesse estudo isso não foi dependente da experiência profissional. Foi objetivo do presente estudo oferecer um instrumento completo e metódico para auxiliar o médico veterinário na investigação clínica do sistema respiratório dos equinos, o que foi alcançado. A avaliação individual de cada avaliador encontra-se nos Quadros 1 e 2.

Quadro 1 - Avaliação qualitativa das sugestões propostas pelos avaliadores (A) 1, 2, 3, 4, 6,7, 8, 9, e 10 em relação ao primeiro e segundo instrumentos de avaliação e conclusões a respeito das avaliações

\begin{tabular}{|c|c|c|c|}
\hline & Sugestões instrumento 1 & Sugestões instrumento 2 & $\begin{array}{c}\text { Conclusões a respeito } \\
\text { das avaliações }\end{array}$ \\
\hline $\mathrm{A} 1$ & $\begin{array}{l}\text { Incluir avaliação de mucosas no } \\
\text { exame geral, dilatação das narinas, } \\
\text { fluxo de ar nasal, linha de esforço } \\
\text { abdominal, avaliação dos seios } \\
\text { nasais, e o termo "dispneia mista". }\end{array}$ & $\begin{array}{c}\text { Colocar o exame geral no início do } \\
\text { exame, logo após a identificação do } \\
\text { animal. O instrumento agora ficou } \\
\text { bem prático. }\end{array}$ & $\begin{array}{l}\text { Considerou-se a avaliação como } \\
\text { positiva, uma vez que todas as } \\
\text { sugestões presentes na análise } \\
\text { do primeiro instrumento foram } \\
\text { cumpridas. }\end{array}$ \\
\hline A2 & $\begin{array}{l}\text { Incluir na avaliação o peso do } \\
\text { animal, coloração da mucosa, } \\
\text { adicionar um espaço maior em } \\
\text { observações e avaliação da bolsa } \\
\text { gutural. }\end{array}$ & $\begin{array}{l}\text { Incluir mais linhas em observações, } \\
\text { se possui exame de mormo, se } \\
\text { há outras manifestações clínicas } \\
\text { sistêmicas, se há algo a mais } \\
\text { relacionado à etiologia dos sinais } \\
\text { clínicos apresentados, incluindo a } \\
\text { avaliação odontológica. }\end{array}$ & $\begin{array}{l}\text { Considerou-se a avaliação } \\
\text { negativa, uma vez que as sugestões } \\
\text { permaneceram no segundo } \\
\text { instrumento de avalição. }\end{array}$ \\
\hline A3 & $\begin{array}{l}\text { Incluir a possível presença de } \\
\text { alimento em secreção nasal. }\end{array}$ & $\begin{array}{l}\text { Adicionar um campo "observações" } \\
\text { em auscultação para melhor } \\
\text { descrição dos achados em cada um } \\
\text { dos hemitórax. }\end{array}$ & $\begin{array}{l}\text { Considerou-se que a avaliação foi } \\
\text { positiva, uma vez que a sugestão } \\
\text { presente na análise da primeira } \\
\text { ficha foi cumprida. }\end{array}$ \\
\hline A4 & $\begin{array}{c}\text { Incluir a linha de esforço abdominal } \\
\text { e disfagia. }\end{array}$ & Sem nenhuma nota & $\begin{array}{l}\text { Considerou-se que a avaliação foi } \\
\text { positiva e que as sugestões foram } \\
\text { atendidas. }\end{array}$ \\
\hline A6 & $\begin{array}{l}\text { Melhorar a disposição das } \\
\text { informações/organização. }\end{array}$ & $\begin{array}{l}\text { O instrumento leva tempo para } \\
\text { ser preenchido, pois é completo, } \\
\text { sendo assim, não tem como ter } \\
\text { preenchimento rápido. }\end{array}$ & $\begin{array}{c}\text { Considerou-se a avaliação positiva, } \\
\text { uma vez que não houveram } \\
\text { sugestões. }\end{array}$ \\
\hline A7 & Sem nenhuma nota & $\begin{array}{l}\text { Incluir o histórico do paciente e um } \\
\text { espaço para avaliação de respiração } \\
\text { forçada. }\end{array}$ & $\begin{array}{c}\text { Considerou-se a avaliação negativa, } \\
\text { uma vez que houveram novas } \\
\text { sugestões no instrumento de } \\
\text { avaliação } 2 \text {. }\end{array}$ \\
\hline A8 & $\begin{array}{l}\text { Incluir como o alimento é oferecido, } \\
\text { se é de forma alta ou baixa. }\end{array}$ & $\begin{array}{l}\text { Instrumento de avaliação completo, } \\
\text { por isso demanda tempo ao seu } \\
\text { completo preenchimento. }\end{array}$ & $\begin{array}{l}\text { Considerou-se a avaliação positiva, } \\
\text { apesar de a avaliadora relatar que } \\
\text { há uma demanda de tempo para o } \\
\text { preenchimento. }\end{array}$ \\
\hline A9 & Sem nenhuma nota & $\begin{array}{c}\text { Por ser um instrumento de avaliação } \\
\text { completo do sistema respiratório, } \\
\text { leva um bom tempo a ser } \\
\text { preenchido. }\end{array}$ & $\begin{array}{l}\text { Considerou-se a avaliação positiva, } \\
\text { com mérito, apesar da demanda de } \\
\text { tempo para o preenchimento. }\end{array}$ \\
\hline A10 & $\begin{array}{l}\text { Incluir espaço para histórico clínico, } \\
\text { maior para observações, adicionar } \\
\text { se o paciente está sob tratamento, } \\
\text { qual a prescrição e quais serão os } \\
\text { exames de sequência. }\end{array}$ & $\begin{array}{c}\text { Incluir o histórico, qual o tratamento } \\
\text { sugestivo, se há um diagnóstico } \\
\text { preventivo, e quais serão os exames } \\
\text { complementares realizados/ } \\
\text { requisitados. }\end{array}$ & $\begin{array}{l}\text { Considerou-se a avaliação neutra, } \\
\text { uma vez que alguns tópicos foram } \\
\text { implementados no instrumento } \\
2 \text { com relação ao campo de } \\
\text { sugestões do } 1 .\end{array}$ \\
\hline
\end{tabular}


Quadro 2 - Avaliação qualitativa das sugestões propostas pelos avaliadores (A) 11,12, 15, 16, 18, 19 e 20 em relação ao primeiro e segundo instrumentos de avaliação e conclusões a respeito das avaliações

\begin{tabular}{|c|c|c|c|}
\hline & Sugestões instrumento 1 & Sugestões instrumento 2 & $\begin{array}{l}\text { Conclusões a respeito } \\
\text { das avaliações }\end{array}$ \\
\hline A11 & $\begin{array}{l}\text { Incluir um espaço para histórico } \\
\text { clínico e para anotar se o animal } \\
\text { está em algum tratamento. Incluir } \\
\text { no exercício tipo e frequência de } \\
\text { exercício. }\end{array}$ & $\begin{array}{l}\text { As divisões de exame ficaram } \\
\text { práticas e completas, porém } \\
\text { poderiam destacar (negrito/caixa } \\
\text { alta) para que nenhum exame passe } \\
\text { sem ser realizado. }\end{array}$ & $\begin{array}{l}\text { Considerou-se a avaliação positiva, } \\
\text { apesar da sugestão de mudança } \\
\text { para melhora visual da análise. }\end{array}$ \\
\hline A12 & Poderiam trocar o termo "normal" & $\begin{array}{l}\text { Incluir o histórico, o exame em } \\
\text { repouso é diferente do exame em } \\
\text { exercício e um espaço maior para } \\
\text { medicação. }\end{array}$ & $\begin{array}{c}\text { Considerou-se a avaliação negativa, } \\
\text { uma vez que houveram mais } \\
\text { sugestões ao instrumento } 2 \text {. }\end{array}$ \\
\hline A15 & Sem nenhuma nota & $\begin{array}{l}\text { Instrumento bom para pacientes } \\
\text { com problema respiratório; não } \\
\text { especifica para outras doenças/ } \\
\text { outros pacientes; falta incluir o } \\
\text { histórico do paciente. }\end{array}$ & $\begin{array}{c}\text { Considerou-se a avaliação positiva, } \\
\text { uma vez que o intuito deste } \\
\text { processo de análise é somente } \\
\text { para pacientes com afecções } \\
\text { respiratórias. }\end{array}$ \\
\hline A16 & Sem nenhuma nota & $\begin{array}{l}\text { Por ser uma ficha do sistema } \\
\text { respiratório, o tempo está } \\
\text { bom, porém como são muitas } \\
\text { informações, a ficha demora a ser } \\
\text { preenchida. }\end{array}$ & $\begin{array}{c}\text { Considerou-se a avaliação } \\
\text { positiva, apesar de ter uma certa } \\
\text { demanda a ser preenchida devido } \\
\text { à necessidade da riqueza de dados } \\
\text { para o diagnóstico. }\end{array}$ \\
\hline A18 & $\begin{array}{l}\text { Incluir as possíveis relações entre } \\
\text { corrimento nasal e alterações } \\
\text { oftálmicas. Informações sobre } \\
\text { exames complementares. }\end{array}$ & $\begin{array}{l}\text { Incluir a relação corrimento nasal/ } \\
\text { alteração oftálmica e informações } \\
\text { de exames complementares. }\end{array}$ & $\begin{array}{l}\text { A avaliação foi igual para ambas } \\
\text { as análises, logo considerou-se a } \\
\text { avaliação neutra. }\end{array}$ \\
\hline A19 & Sem nenhuma nota & $\begin{array}{l}\text { Ficha clara, limpa e fluida, prática de } \\
\text { ser respondida. }\end{array}$ & $\begin{array}{l}\text { Considerou-se a avaliação positiva, } \\
\text { uma vez que houveram elogios à } \\
\text { segunda forma de análise. }\end{array}$ \\
\hline A20 & $\begin{array}{l}\text { Incluir alterações pertinentes a } \\
\text { pleurodinia. }\end{array}$ & $\begin{array}{l}\text { Poderia conter mais informações } \\
\text { como dificuldade de locomoção, } \\
\text { aumento do ruído/tosse. }\end{array}$ & $\begin{array}{l}\text { São sugestões que poderão ser } \\
\text { acatadas para as próximas versões } \\
\text { do processo de análise respiratória. }\end{array}$ \\
\hline
\end{tabular}

Os avaliadores 13, 14 e 17 não adicionaram nenhum comentário ao campo sugestões, em nenhuma das duas avaliações. Isto pode ser visto de forma positiva, uma vez que não propuseram sugestões para melhorias.

O instrumento de avaliação clínica é um documento de direcionamento e registro de cada paciente, que serve para o armazenamento de todas as informações clínicas, ambientais, nutricionais e histórico do paciente em questão. De acordo com - Conselho Federal de Medicina Veterinária - CFMV (Brasil, 2020), o médico veterinário tem o dever de manter seus prontuários por cinco anos, contendo todos os dados de avaliações e tratamentos desde o último atendimento, mesmo em caso de óbito. As informações constantes são de direito do cliente, além de poder ser necessário em eventual questão judicial. Assim, além de servir como orientação para o médico veterinário, também serve para a sua segurança, mantendo sua conduta registrada.

Considerando-se os comentários deixados no campo de sugestões (Quadros 2 e 3), o fato de o instrumento 2 não reduzir o tempo para o preenchimento em relação ao 1 pode ser pela maior quantidade de informações incluídas no instrumento 2. Além disso, não pretendeu-se encurtar o exame clínico respiratório, mas, pelo contrário, buscou-se o instrumento mais completo, procurando facilitar 
o preenchimento e ter um registro mais claro de informações. Nesse sentido, incluiu-se ilustrações da face para a observação e percussão dos seios paranasais. Adicionalmente, incluiu-se as divisões dos hemitórax, local para informações sobre a queixa principal e anamnese detalhada, incluindo medicações com doses anteriores e o ambiente onde o animal convive (McGorum, 2007; Ivester et al., 2014; Wilson e Lakritz, 2014).

Sendo assim, o intuito deste processo de análise é única e exclusivamente avaliar o sistema respiratório equino após queixa ou observações iniciais indicativas de envolvimento desse sistema durante o exame físico geral. Não objetivou-se incluir avaliações complementares e que pudessem envolver outros órgãos e sistemas. Desta forma, o instrumento inicia pela queixa do proprietário, seguido pela avaliação do tipo de alimentação e de ambiente em que o paciente vive, manejo geral e anamnese, passandose ao exame físico geral (Davis, 2018).

\section{Conclusão}

No presente estudo foi possível obter um instrumento de avaliação do sistema respiratório de equinos mais detalhado, claro e intuitivo para médicos veterinários experientes na área e também para generalistas. A partir dos resultados obtidos, esse instrumento ainda precisa ser validado por um número maior de médicos veterinários para confirmar os presentes resultados, assim como comprovar sua praticidade.

\section{Agradecimentos}

Os autores gostariam de deixar seus agradecimentos a todos os avaliadores presentes na análise, sendo eles: Alan Cortese, Alessandra Franco Cechim, Alessandro Mercadante, Ana Paula Rezende Silva, Andressa Duarte Lorga, Anny Gomes, Gilson Pedro Amaral Filho, Henriette Graf, Jessica do Rocio Janiszewski, Júlio David Spagnolo, Lucimara Strugava, Luis Felipe P. dos Passos, Luis Fernando Bastos, Manuella Camarotti, Marina Silvestre Vidal, Natália Perboni Michelotto, Stefano Strano Calomeno, Tatiana Yumi e Vanessa Alberini.
Os autores também agradecem à Pontifícia Universidade Católica do Paraná (PUCPR) pelo apoio financeiro à pesquisa através de bolsa de estudos.

\section{Referências}

Albrecht B, Aven M, Lamar J, Ingo Lang I. Ciclesonide for the treatment of airway disease in horses. U.S. Patent; 2018 [acesso 31 out 2020]. Disponível em: https://patents.google.com/ patent/US10441597B2/en

Brasil. Conselho Federal de Medicina Veterinária. Resolução $n^{\circ}$ 1321, de 24 de Abril de 2020. Institui normas sobre os documentos no âmbito da clínica médico-veterinária e dá outras providências. Brasília: Diário Oficial da União; 27 abr 2020.

Christley RM, Hodgson DR, Rose RJ, Hodgson JL, Wood JLN, Reid SW. Coughing in thoroughbred racehorses: risk factors and tracheal endoscopic and cytological findings. Vet Rec. 2001;148(4):99-104.

Couëtil LL, Cardwell JM, Gerber V, Lavoie JP, Léguillette R, Richard EA. Inflammatory airway disease of horses - revised consensus statement. J Vet Intern Med. 2016;30(2):503-15.

Davis E. Disorders of the Respiratory System. In: Reed SM, Bayly WM, Sellon DC, editores. Equine internal medicine. 4 ed. Saint Louis: Saunders Elsevier; 2018. p. 323-86.

Fernandes WR, Sanchez A, Ramos MCC, Souza VRC, Coelho CS. Microbiological fidings of tracheobronchial washes of healthy horses and those with respiratory diseases. Ars Vet. 2011;27(2):73-9.

Hoffman AM. Inflammatory airway diseases: definitions and diagnosis in the performance horse. In: Robinson NE, editor. Current therapy in equine medicine. 5 ed. St Louis: Saunders; 2003. p. 412-7.

Ivester KM, Couëtil LL, Moore GE, Zimmerman NJ, Raskin RE. Environmental exposures and airway inflammation in young thoroughbred horses. J Vet Intern Med. 2014;28(3):918-24.

Kusano K, Hobo S, Ode H, Ishikawa Y. Tracheal endoscopic and cytological findings and blood examination results in thoroughbred racehorses suspected to have lower respiratory tract disease. J Equine Sci. 2008;19(4):97-102. 
Kutasi O, Balogh N, Lajos Z, Nagy K, Szenci O. Diagnostic approaches for the assessment of equine chronic pulmonary disorders. J Equine Vet Sci. 2011;31(7): 400-10.

Léguillette R, Tohver T, Bond SL, Nicol JA, McDonald KJ. Effect of dexamethasone and fluticasone on airway hyperresponsiveness in horses with inflammatory airway disease. J Vet Intern Med. 2017;31(4):1193-201.

McGorum BC, Dixon PM. Clinical examination of respiratory tract. In: McGorum BC, Dixon PM, Robinson NE, Schumacher $\mathrm{J}$, editores. Equine Respiratory Medicine and Surgery. Philadelphia: W.B. Saunders; 2007. p. 103-17.
Robertson JT. Pharynx and larynx. In: Beech J, editor. Equine respiratory disorders. Philadelphia: Lea e Febiger; 1991. p. 331-88.

Seahorn TL, Beadle RE. Summer pasture-associated obstructive pulmonary disease in horses: 21 cases (1983-1991). J Am Vet Med Assoc. 1993;202(5):779-82.

Wilson WD, Lakritz J. Alterations in Respiratory Function. In: Smith B. Large animal internal medicine. 5 ed. Saint Louis: Mosby; 2014. p. 40-75. 